\title{
Analysis of the Degradation of $\mathrm{BaTiO}_{3} \underline{\text { Resistivity }}$
}

\section{due to Hydrogen Ion Incorporation: Impedance Spectroscopy and Diffusion Analysis}

\author{
Damoon Sohrabi Baba Heidary, Clive A. Randall*
}

Center for Dielectrics and Piezoelectrics, Materials Research Institute, the Pennsylvania State University, University Park, Pennsylvania 16802, USA

\section{Corresponding Author: Clive A. Randall}

(814) 863-1328, car4@psu.edu

\begin{abstract}
The resistivity of insulator oxide materials can be compromised when they are exposed to hydrogen gas at high temperatures, as insulation resistance can significantly decrease. In this study, we differentiate between electrode, grain boundary, and grain contributions to total resistivity by impedance spectroscopy (IS) tests and a $3 \mathrm{RC}$ model. It turns out that the largest contribution to total resistivity comes from electrode Schottky barriers, which control the major part of the degradation. Based on the IS analysis, the hydrogen diffusion coefficients of those three components were successfully calculated and compared with the diffusion coefficient in other systems. Determination of the hydrogen diffusion in grains and grain boundaries is important in understanding how hydrogen penetrates to capacitors and can also be useful for applications that involve extreme environments. In this study, we also considered the kinetics and role of the metal electrode chemistry $(\mathrm{Ag}, \mathrm{Au}$, and $\mathrm{Pt})$ and the thickness of active layers on the hydrogen degradation.
\end{abstract}

Keywords: hydrogen diffusion; resistivity degradation; impedance spectroscopy; $\mathrm{BaTiO}_{3}$; grain boundary

\section{Introduction}


Diffusion of hydrogen ions into electronic materials can cause the degradation of their electrical insulation properties. The piezoelectric degradation of $\mathrm{Pb}(\mathrm{Zr}, \mathrm{Ti}) \mathrm{O}_{3}(\mathrm{PZT})$ due to hydrogen cations is well known and has been studied by many researchers[1-4]. For example, PZT can be used as an actuator in hydrogen internal combustion engines as a better replacement for port injections, but because of hydrogen degradation, they fail frequently $[5,6]$. Furthermore, hydrogen can be undesirable in ferroelectric random access memory; hydrogen ions tend to bind with oxygen atoms and hinder ferroelectric hysteretic switching in PZT[4]. Hydrogen can also decrease resistivity and change capacitance in PZT [7]. Furthermore, the perovskite oxides $\left(\mathrm{ABO}_{3}\right)$ coated on a silicon wafer should be annealed at $450^{\circ} \mathrm{C}$ in the reductive atmosphere, containing hydrogen gas, to prevent $\mathrm{SiO}_{2}$ formation at the interface [8]. One concern is that hydrogen ions may diffuse to the perovskite layer and degrade its resistivity [9].

On the other hand, hydrogen diffusion in perovskite oxides can be considered a desirable phenomenon. Since perovskite is one of the most stable crystal structures, it can be used as a separator material in electrochemical cells[10,11].

Numerous techniques can be found in literature for measuring hydrogen diffusion in perovskite oxides. Those techniques can be as simple as exposing samples to hydrogen and watching the color change from the exposed surface toward the virgin area[12] or using more advanced techniques, such as impedance spectroscopy $[10,13]$, thermal desorption studies (TDS) [14], quasi-elastic neutron scattering (QENS)[15], or proton nuclear magnetic resonance (NMR)[5]. The reported diffusion coefficient for the same material can be different in different papers. Those differences can be due to the fact that some perovskite oxides change their crystal structure by temperature, and the diffusion energy barrier may increase by the reduction of crystal symmetries [10]. Some instrumental differences can lead to measuring different diffusion coefficients; for example, in the impedance techniques, the electrodes can 
act as a catalyst and facilitate hydrogen ionization [16], so one may measure rates with different electrodes.

Impedance spectroscopy has been proven to be a powerful technique to differentiate between the contributions of different components in total impedance [17]. There are several methods to analyze the measured data in impedance spectroscopy tests [18]. For simple systems, the method of equivalent circuit is popular[19,20]. The objective of this work is to use the impedance changes and the equivalent circuit analysis to provide a more detailed understanding of resistivity changes and infer routes for $\mathrm{H}$-ion diffusion into the microstructure.

\section{Experimental Procedures}

$\mathrm{BaTiO}_{3}$ powders co-doped with $\mathrm{Y}_{2} \mathrm{O}_{3}-\mathrm{MnO}$ X7R formulations were tape casted into layers with a thickness of 50 and $20 \mu \mathrm{m}$ and cut into $1 \times 1$ inch squares. For buried base-metal electrode (BME) samples, the electrodes patterns, $4 \times 5$ rectangles with the dimensions of $2 \times 4.5 \mathrm{~mm}$, were printed with a homemade nickel ink (made with Sheoi Chemical Ni powder) on the two layers with 20- $\mu$ m thickness. Tapes and electrodes were aligned so that every rectangle had an overlap area of $2 \times 2.5 \mathrm{~mm}$ with an extent of $1 \mathrm{~mm}$ in both sides in its length direction, see the "exposed electrodes" face in Figure 1. After a satisfactory alignment, those $20 \mu \mathrm{m}$ layers were stacked with six $50-\mu \mathrm{m}$ squares on the top and the bottom and were then laminated and cut into separate rectangles with a pair of buried electrodes. Next, the samples were sintered at $1300^{\circ} \mathrm{C}$ for 2 hours in $10^{-10}$ atm of oxygen partial pressure, followed by reoxidation at $800^{\circ} \mathrm{C}$ in $10^{-8}$ atm of oxygen partial pressure for 8 hours. The prototyped capacitors had one active layer with $18 \mu \mathrm{m}$ thickness, with high quality dielectric performance. To make sure hydrogen would be exposed in the same way to the active layer in all the samples, they were cut along the electrode in one side, as shown in Figure 1 as "exposed electrodes". 
The other type of sample was monolithic structures, sometimes called k-squares. They were made by stacking tape casted $\mathrm{BaTiO}_{3}$ layers to get a $550 \mu \mathrm{m}$ thickness, then laminated and cut to $7 \times 7 \mathrm{~mm}$ thickness with Universal M360 laser cutter. Next, these were sintered in the above mentioned process. Then the samples were washed, first with acetone, and then isopropanol, for $10 \mathrm{~min}$ in a ultrasonic sonic bath, followed by drying in an oven at $120^{\circ} \mathrm{C}$ for 20 minutes. Next, the electrodes with the same area as BME samples were applied to both sides of the samples with Quorom EMS 150 Sputter Coater. To make sure the electrodes are in the center and face each other, a label was cut with the laser cutter to a $7 \times 7$ $\mathrm{mm}$ square, with a circle in the middle and an area of $5 \mathrm{~mm}^{2}$, and used as a mask in the electrode sputtering.

Impedance spectroscopy was executed with SR-830-DSP Lock-in connected to homemade charge measurement hardware. An HP4284A LCR meter (Hewlett-Packard) was used to measure impedance at $1 \mathrm{kHz}$. A PSU constructed furnace, inside which was a sealed stainless steel box with 4 sample fixtures, was used to heat the samples up to $255^{\circ} \mathrm{C}$. A pipe was connected to the box to blow in reducing gas into the sample chamber with adjustable rates from 0 to $500 \mathrm{cc} / \mathrm{min}$. Forming gas ( $5 \%$ hydrogen and $95 \%$ nitrogen) was used to expose the samples to hydrogen gas. For convenience, the forming gas will be referred to as hydrogen atmosphere from now on. A K-type thermocouple was placed inside the box to make sure the true temperature would be measured during the tests.

To be sure about the precision of electrical measurements, the hardware was tested with NIST traceable air gap standard capacitors from HP. In addition, the $1 \mathrm{nF}$ standard capacitor was tested with Solartron ModuLab XM as well and there was an excellent match between two results.

TEM samples were prepared by FEI-Quanta-200 FIB (Oregon, USA). TEM micrographs were done by Philips 420.

\section{Results and Discussion}




\subsection{Impedance Spectroscopy}

Impedance spectroscopy has previously been applied to base metal capacitors to assess interfacial changes under typical time dependent breakdown, re-oxidation, and ambipolar diffusion for dielectric /piezoelectric based materials [19,21,22]. Here we consider, in a similar way, the effects of $\mathrm{H}$-ion degradation. The impedance measurements were made between frequencies 0.01 and $10^{5} \mathrm{~Hz}$ at $0.1 \mathrm{~V}$ and across a temperature range of 215 and $255^{\circ} \mathrm{C}$. $3 R C$ model was applied to interpret the raw ColeCole plots in Figure 3 (a). A 3RC model was chosen, because there are three components in every capacitor (electrode and grain boundary Schottky barriers, and bulk grains), which have separate contributions to total resistivity and capacitance. Plus, the quality of the fitting with an average of $\mathrm{R}^{2}=0.98$ between measured data and fitting curves provides another support for using the $3 \mathrm{RC}$ model. Furthermore, the other researchers used the $3 R C$ model to successfully interpret their raw data for the same type of capacitors $[19,20]$. However, if there is a core-shell dominated structure in capacitors, it may limit 3RC model [20]. A transmission electron microscopy (TEM) diffraction contrast analysis was executed on the samples in order to search for the core-shell structure. As shown in Figure 2, core-shell is not the dominant structure in the current samples. The calculated activation energies for grain $(0.78$ $\mathrm{eV})$, grain boundary $(1.12 \mathrm{eV})$, and electrode $(1.15 \mathrm{eV})$, based on an Arrhenius relationship, are also comparable to similar studies $[19,20]$.

We are able to infer the respective microstructural contribution to each impedance element through the guidance of the previous works. The differentiation between bulk grains and Schottky barriers is relatively easy; grains have the highest slope in $1 / C_{i}$ vs temperature (where $C_{i}$ is capacitance of $i^{\text {th }}$ element) curves $[19,20]$. Those curves are shown in Figure $3(b)$, and the steepest slope belongs to grains. 
Figure 3 (c) shows the resistivity of the three components; the electrode has the highest resistivity in all temperatures. Considering the fact that there are many grain boundaries in the active layer, but their combined resistivity is still less than electrodes, one can conclude the electrode barrier height should be much higher than that of the grain boundaries, as schematically shown in Figure 4. Electrodes have Schottky barriers, and the grain boundaries have back-to-back Schottky barriers in the capacitors. One can consider an impedance element, which includes in parallel one ideal resistor and one ideal capacitor, for every grain, grain boundary, and electrode in Figure 4. Then all similar types of impedance are added together and make one of the RC's in the 3RC model.

The samples were then exposed to a hydrogen atmosphere at $245^{\circ} \mathrm{C}$, and the impedance spectroscopy was executed repeatedly for five hours; between every IS, the impedance was measured for 15 minutes at $1 \mathrm{kHz}$ by LCR meter. As shown in Figure $5(\mathrm{a})$, there is a good match between both curves from the experimental data, obtained by LCR meter, and the ones from 3RC model. That match can be another support for the validity of the $3 R C$ model. The Cole-Cole plots are shown in Figure 5 (b) in relation to the changes with hydrogen exposure. The total impedance reduces as the hydrogen ions enter the dielectric as interstitial donors, as would be anticipated from prior studies $[7,23,24]$.

As shown in Figure 5 (c), the maximum reduction in the resistivity occurs at the electrodes and with much less amplitude in grain boundaries; the resistivity reduction in the grain component is almost negligible. Hydrogen ions may decrease $\mathrm{BaTiO}_{3}$ resistivity with two mechanisms. First, hydrogen gas $\left(\mathrm{H}_{2}\right)$ can be ionized according to Eq. (1), and as a donor gives free electrons to conduction band, so absorbing hydrogen generally decreases resistivity.

$$
\mathrm{H}_{2} \rightarrow 2 \mathrm{H}_{i}+2 e^{-}
$$


More importantly, hydrogen ions can pile up in the interfaces (electrode-dielectric or grain boundary) and reduce the depletion width, causing Schottky barriers to have lower resistance [25][26]. There is an order of magnitude reduction in the electrode resistivity that is much higher than the grain boundary reduction, which can be due to the fact that the largest contribution to the total resistivity comes from electrodes, and its reduction would be the largest portion of resistivity reduction and main cause of the total resistivity degradation. The proposed mechanism for hydrogen ion diffusion and distribution is schematically shown in Figure 6. This is analogous to observations by Opitz et al. [27] with tracer oxygen diffusion, which has been proposed to account for reoxidation kinetics. The hydrogen gas dissociates into $2 \mathrm{H}^{+}$cations and easily migrates along the electrode-dielectric interfaces and grain boundaries, and later diffuses into the grains.

\subsection{Diffusion Coefficient}

To describe the hydrogen diffusion into the samples, the boundary conditions should be correctly defined, then the concentration distribution can be calculated by the second Fick's Law [28].

The active layer was defined as a one dimensional system along the electrodes, started from the exposed surface toward the inside of the sample, as shown in Figure 1 and Figure 6. This modelling approach makes sense, since the amount of hydrogen in the active layer affects the amount of measured resistivity in impedance spectroscopy tests, and the closest way for hydrogen ions enter to the active layer is through the exposed surface. This experimental set up is similar to that used by Donnelly and Randall for calculating oxygen diffusion in $\mathrm{BaTiO}_{3}$ and lead oxide volatility in PZT piezoceramics $[13,22,29]$.

The system may be considered as semi-infinite media with the following boundary condition at the exposed surface: 


$$
\frac{d N(t)}{d t}=\alpha\left(C_{0}-C_{S}\right)
$$

where $C_{0}$ and $C_{S}$ are concentration of hydrogen in atmosphere and on an exposed surface, respectively; $\mathrm{N}$ is the total hydrogen concentration in the active layer, and $\alpha$ is a proportionality constant [30]. In other words, Eq. (2) simply shows that the entering rate of total hydrogen ions inside the active layer is proportional to the concentration difference between atmosphere and surface.

Next, the measured resistivity should be related to hydrogen ion concentration at the electrode. The impedance tests and $3 R C$ model indicate that the electrode contribution to the total resistivity and its reduction by time are experimentally accessible, see Figure 5 (c). Inverse of resistivity or conductivity $(\sigma)$ can be related to leakage current density $(\mathrm{J})$, and then $\mathrm{J}$ can be related to hydrogen concentration through thermionic and field emission conductivity mechanisms for electrode Schottky barriers, which was studied in a previous paper [26]. Here, for simplicity, a Taylor series is used to relate the conductivity $(\sigma)$ to the Hydrogen concentration. Just the first four terms of the Taylor series are used, and later it will be shown that the third and fourth terms are too small and negligible. The Taylor series would be as:

$$
\sigma=A+B N+C N^{2}+E N^{3}
$$

where $\mathrm{A}, \mathrm{B}, \mathrm{C}$, and $\mathrm{E}$ are constant and $\mathrm{N}$ is total hydrogen concentration in front of electrodes.

Now the second Fick's equation in semi-infinite media for the boundary condition in Eq. (2) may be solved as:

$$
N(t)=\left(\frac{C_{0}}{h}\right)\left[\exp \left(h^{2} D t\right) \operatorname{erfc}(h \sqrt{D t})-1+\frac{2}{\sqrt{\pi}} h \sqrt{D t}\right]
$$


Where: $t$ is time, $D$ diffusion coefficient, and $h=\frac{\alpha}{D}$.

The proof of Eq. (4) is given in Ref. [30]. Now, Eq. (3) and (4) can be used to relate resistivity (the inverse of conductivity) to time; on the other hand, there are resistivity-time curves, resultant from impedance spectroscopy tests for the electrode contribution. For example, see Figure 5 (c). By using a least square method and a Matlab code, one would be able to fit the curves with the equations and find the amount of $A, B, C, E, \alpha$, and $D$. The best fitting curve for the electrode resistivity vs. time, shown in Figure 7 (a), is labeled as "Electrodes". The fitting has R-square of 0.9915. C and E, both negligible compared to the measured conductivities, and Eq. (3) would be:

$$
\sigma=4.35 \times 10^{-9}+7.15 \times 10^{-5} N
$$

The diffusion coefficient for electrodes at $245^{\circ} \mathrm{C}$ is shown in Table 1 . Based on the Figure 6, one can assume the same governing equation and boundary conditions as electrodes for grains and grain boundaries. By using their resistivity, resultant from impedance spectroscopy, diffusion coefficient in these components at $245^{\circ} \mathrm{C}$ can be found. The curve fittings for grains and grain boundaries are shown in Figure 7 (a), and R-squares are 0.998 and 0.991, respectively. Those curves were again obtained based on the linear relation between conductivity and hydrogen concentration. The diffusion coefficients are shown in Table 1. Since the diffusion coefficients are highest at the dielectrics-electrodes interface, the hydrogen can diffuse rapidly through that interface. The second highest diffusion coefficient belongs to the grain boundaries, and the minimum with much lower value to grains. Thus, the schematic drawing in Figure 6 is a reasonable representative of the diffusion regime, discussed by Mehrer [31]. Since electrode has the highest diffusion coefficient, it is not surprising that the highest hydrogen ion 
concentrations are calculated in front of electrodes. However the concentrations are small and in the order of ppm.

By knowing the diffusion coefficients (D) and $\mathrm{h}$ in Eq. (4), one can calculate the estimated distribution of hydrogen ions along the electrode- $\mathrm{BaTiO}_{3}$ interface $(\mathrm{x})$, shown in Figure 6 , by using Eq. (6):

$$
\frac{C(x, t)}{C_{0}}=\operatorname{erfc}\left(\frac{x}{2 \sqrt{D t}}\right)-\exp \left(h x+h^{2} D t\right) \operatorname{erfc}\left(\frac{x}{2 \sqrt{D t}}+h \sqrt{D t}\right)
$$

where $C(x, t)$ is the concentration of hydrogen in respect to position $(x)$ and time $(t)$. For clarification, Eq.(7) shows the relation between $N(t)$ and $C(x, t)$.

$$
\frac{d N(t)}{d t}=-D \frac{\partial C(x=0, t)}{\partial x}
$$

In the other words, one can think of $\mathrm{N}(\mathrm{t})$ as the area under the $\mathrm{C}(\mathrm{x}, \mathrm{t})$ curves, shown in Figure $7(\mathrm{~b})$.

The amount of hydrogen increases rapidly in the first several micrometers of the interface, as seen in Figure $7(b)$. For example, there is a rapid increase of hydrogen concentration in front of the electrode in the first 1.5 hours. However, the rate of proton concentration increase is reduced by passage of the time; for example, there is not much difference between the curves for 9 and 10.5 hours. This rapid increase manifests itself in a large drop in the electrode resistivity in Figure 5 (c) and is the key to explain the fast resistivity degradation in the capacitors, as discussed in the previous paper [26].

The same impedance tests were repeated at 275 and $305^{\circ} \mathrm{C}$, and then by the above mentioned procedure, the diffusion coefficients for the electrodes, grain boundaries, and grains were calculated. Since diffusion is a thermally activated process, there is an Arrhenius relationship between $D$ and $T$ as follows [26]: 


$$
D=D_{0} \exp \left(\frac{-E_{a}}{k T}\right)
$$

where $k$ is Boltzmann constant, $T$ temperature, $D_{0}$ pre-exponential, and $E_{A}$ activation enthalpy. The log of the calculated diffusion coefficients vs. reciprocal temperature is shown in Figure 7 (c). As seen, the diffusion coefficient is highest for the electrode interface at every temperature. Diffusion coefficients in grain boundaries are slightly lower, but in grains are much lower than in electrodes. From Figure 7 (c), one can obtain the pre-exponential factor $\left(D_{0}\right)$ and activation enthalpy $\left(E_{A}\right)$ for hydrogen diffusion in the three components, as shown in Table 1.

The rate of hydrogen successful jump from one oxygen atom to another, shown by $\omega$, may be written as [31,32]:

$$
\omega=v_{0} \exp \left(-\frac{S_{a}}{k}\right) \exp \left(-\frac{E_{a}}{k T}\right)
$$

where $v_{0}$ is an attempt frequency, and $S_{a}$ entropy of activation. $S_{a}$ and $E_{a}$ are related to activation energy $\left(G_{a}\right)$ by :

$$
G_{a}=E_{a}-T S_{a}
$$

Since $D$ is proportional to $\omega$, one can expect that there would be a relation between $D_{0}$ and $E_{a}$. Obtaining that relationship is complicated for hydrogen and needs a quantum mechanical approach [31]. For example, Kreuer $[10,11]$, by using quantum molecular dynamics, successfully calculated the bulk hydrogen diffusion coefficient in several perovskite oxides. For comparison to the present paper, $E_{a}$ and $D_{0}$ for several perovskite oxides for diffusion in bulk (grains) are given in Table 2. As seen, both $E_{a}$ 
and $D_{0}$ would change with different constituent atoms, dopant, or dopant concentration, but the range stays between ( 0.17 to $0.63 \mathrm{eV})$ and $\left(3 \times 10^{-5}\right.$ to $\left.3.47 \times 10^{-2} \mathrm{~cm}^{2} / \mathrm{s}\right)$, respectively. The reported $E_{a}$ and $D_{0}$ in Table 1 for grains lie within those bounds. Moreover, Norby [33], by considering $E_{a}$ in around 35 perovskite oxides $\left(\mathrm{ABO}_{3}\right)$, found the following empirical relationship between electronegativity difference of $\mathrm{A}$ and $\mathrm{B}\left(\Delta \mathrm{X}_{B-A}\right)$ with $\mathrm{E}_{\mathrm{a}}$ :

$$
E_{a}(e V)=4.15 \Delta \mathrm{X}_{B-A}-1.87
$$

Although there is a large scattering in the reported data that they used to find Eq. (11), and the equation does not take the dopant concentration into account, it is a good guide for a rough estimation for $E_{a}$. Plus, the reported activation energies by Norby [33] cover the range of 0.21 to $1.87 \mathrm{eV}$, which almost contain the reported activation energy in Table 2.

Norby and his coworkers [33] briefly reviewed the hydrogen diffusion in grain boundaries; they speculate that the activation energy should be higher in grain boundaries than in grains. There is not yet a comprehensive study on the hydrogen diffusion in the grain boundaries and electrode-dielectrics interface in perovskite oxides to compare with the diffusion coefficients in the present paper. However, they can be rationalized by comparison to the studies on other systems. Naundorf $[38,39]$ reviewed grain boundary diffusion and proved that diffusion in grain boundaries is similar to diffusion in amorphous materials. Kirchheim $[40,41]$ shows that there is a spectrum of enthalpies in amorphous PdCu-Si alloy with a Gaussian distribution due to the disordered structure of amorphous materials.

One can extend the logic in Naundorf's [38,39] and Kirchheim's [40,41] research to the present study. Since there is a spectrum of enthalpies at interfaces and grain boundaries, and hydrogen ions prefer to go to the lower level energy, they fill the lower enthalpy level first; this is why the activation enthalpy $E_{a}$ is higher at the electrode- $\mathrm{BaTiO}_{3}$ interfaces and grain boundaries than in grains (see Table 1). Especially 
in low hydrogen concentration, that enthalpy spectrum can make a large difference [40]. All in all, the diffusion coefficients in interfaces and grain boundaries due to having larger $D_{0}$ are higher than the diffusion coefficients in grains, as shown in Figure 7.

\subsection{Impact of different parameters on resistivity degradation}

Now, by knowing the impedance spectroscopy results and hydrogen diffusion in those RC components, one would be able to check the validity of $3 R C$ model and get a better understanding about hydrogen diffusion by manipulating some parameters in the impedance tests. Two parameters of electrode chemistry and active layer thickness were changed and then checked to determine whether the 3RC model would be able to justify the new results.

\subsubsection{The impact of active layer thickness}

The active layer in BME samples increases from 18 to $500 \mu \mathrm{m}$ in k-square samples. Although the electrode materials and area remains same, the resistivity components in $3 R C$ model increase $5.8,7.7$, and 13.2 times in electrode $(E)$, grain $(G)$, and grain boundary $(G B)$ components, respectively. There is an increase in $G$ and GB resistivity, because the number of grains and GB Schottky barriers increase. The $E$ resistivity increases as well, because the increase in GB and G resistivities leads to a larger voltage drop, and that means less voltage difference at electrode Schottky barriers and eventually less leakage current due to a less contribution of Field Emission conductivity [26].

The resistivities of electrode, grain boundary, and grain extracted from impedance tests by the $3 R C$ model are shown in Figure 8 for BME and k-square samples. K-square results are normalized with the above mention factors, so that every RC component's curve starts from the same place. As can be seen, the amount of degradation is higher in every BME component than in k-square's. Since the active layer is thinner in BME when exposed to the same hydrogen atmosphere, the accumulation of hydrogen ions would be higher in a small portion of BME samples than in k-square ones. The higher hydrogen 
concentration manifests in higher reduction in resistivity. For instance, the BME electrode shows much higher degradation than the k-square, mostly because that hydrogen concentration in front of the ksquare electrode is less accumulated. Since the diffusion coefficient in grain boundary is almost as high as in an electrode interface, as shown in Figure 7 (c), the grain boundaries can be a free path for hydrogen atoms and move them away from the electrode to the inside of active layer in k-squares; regarding the higher thickness of k-square samples, that leads to less hydrogen concentration in front of the electrode. In short, hydrogen ions reach higher concentration in BME electrode, and this is why they are degraded more.

\subsubsection{The impact of electrode materials}

In the case of an ideal Schottky barrier at the electrode interface, in which there is no gap between semiconductor and electrode and no interfacial states to pin the Fermi Level, the difference between metal work function $\left(\phi_{m}\right)$ and semiconductor affinity $(\chi)$ is the Schottky barrier height $\left(\phi_{B}\right)[42]$ :

$$
\phi_{\mathrm{B}}=\phi_{\mathrm{m}}-\chi
$$

So in principle, different electrode metals with different work functions can change the height of Schottky barriers in the absence of pinning. Work function depends on surficial parameters, such as surface cleanliness and surface crystal plane, so one may find different values for work function of a metal in literatures $[43,44]$. Three electrodes of $\mathrm{Ag}, \mathrm{Au}$, and Pt are selected with work functions of 4.26 [45], 5.40 [46], and $5.64 \mathrm{eV}$ [47] in polycrystalline form. The advantages of using those electrodes are, first, their works functions have been well studied and known; second, they all could be easily coated on $\mathrm{BaTiO}_{3}$ k-square samples with the same method (sputtering) to make sure their quality is somewhat similar; third, regarding $\mathrm{BaTiO}_{3}$ electron affinity, $3.9 \mathrm{eV}$ [48], the Schottky barriers are different enough to make tangible changes in IS results [49]. 
The impedance tests were executed on the three samples, and the results are shown in Figure 9. As was expected, a much smaller curve was obtained for virgin sample with silver electrode than the samples with $\mathrm{Au}$ and Pt electrode in Figure 9 (a). As a matter of fact, Ag electrode Schottky barrier is negligible. Pt electrodes shows the largest resistivity, and Au electrode have the second largest resistivity, as expected from electrode work functions and Eq. (12).

In regard to the impact of resistivity changes in the presence of hydrogen gas with different metal electrode chemistries, there is a $35 \%$ reduction in Pt electrode resistivity, while resistivity reduces by $29 \%$ in an Au electrode. It can be concluded that Pt electrode is a better catalyst for hydrogen ionization, given in Eq. (1), than Au. This conclusion is in agreement with earlier observations of Han and Ma [16]. They studied the hydrogen influence on $\mathrm{SBT}\left(\mathrm{SrBi}_{2} \mathrm{Ta}_{2} \mathrm{O}_{9}\right)$ remnant polarization; based on their results, $\mathrm{Pt}$ can pump much more hydrogen to SBT than Au.

All the samples show the same grain resistivity in Figure 9 (b), since they all have the same thickness. The grain boundary resistivity of Pt is slightly higher than $\mathrm{Ag}$ and $\mathrm{Au}$ at the beginning of the exposure; it is due to the higher Schottky barrier in the $\mathrm{Pt}^{-} \mathrm{BaTiO}_{3}$ interface, which leads to a higher voltage drop at interface. However, hydrogen degradation reduces electrode resistivity, which causes a voltage drop decrease at the interface, so that all grain resistivities would be same after the $2^{\text {nd }}$ hour of exposure.

The obvious conclusion from Figure 9 is that the RC contribution for electrodes has been chosen correctly, since it was illustrated that electrode resistivity contribution would experience the maximum change by changing the electrode metals.

\section{Conclusions}

In this paper, the resistivity degradation of $\mathrm{BaTiO}_{3}$ due to hydrogen gas was studied by using impedance spectroscopy. Since there are three main contributors to samples' resistivity (electrode, grain boundary, 
grains), a $3 R C$ model was found to be the best model to fit the Cole-Cole plots, resultant from the impedance tests. Plus, some techniques have been illustrated to differentiate between RC's.

Electrodes have the largest contribution to the resistivity of BME samples when the samples are exposed to hydrogen gas; the largest resistivity reduction also happen at electrodes.

Moreover, hydrogen diffusion coefficients could be successfully calculated, assuming the right boundary conditions and relating measured resistivity to hydrogen concentration at grain, grain boundary, and electrode interface. The pre-exponential factor $\left(D_{0}\right)$ and activation enthalpy $\left(E_{a}\right)$ were respectively measured $2.02 \times 10^{-3} \mathrm{~cm}^{2} / \mathrm{s}$ and $0.52 \mathrm{ev}$ for grains, which are in a good agreement with other reports. The diffusion coefficients at electrode interfaces and grain boundaries are larger than grains. The results were rationalized by findings in other systems. The resultant diffusion coefficients can be useful in fuel cell studies, where people are interested in measuring hydrogen diffusion coefficient in perovskite oxides.

Finally, the effective parameters in impedance measurement are manipulated and show how they affect hydrogen diffusion. In short, the geometry of the active layer influences the degradation; there would be more proton accumulation in BME capacitors than in k-square ones. Electrode chemistry can also affect the total resistivity and the rate of degradation.

\section{Acknowledgements}

This material is based upon work supported by the National Science Foundation, as part of the Center for Dielectrics and Piezoelectrics under Grant No. IIP- 1361503 and 1361571.

\section{References}

[1] Shimakawa Y, Kubo Y. Appl Phys Lett 2000;77:2590.

[2] Lee JK, Park Y, Chung I. J Appl Phys 2002;92:2724. 
[3] Ikarashi N. Appl Phys Lett 1998;73:1955.

[4] Aggarwal S, Perusse SR, Tipton CW, Ramesh R, Drew HD, Venkatesan T, Romero DB, Podobedov VB, Weber a. Appl Phys Lett 1998;73:1973.

[5] Alvine KJ, Vijayakumar M, Bowden ME, Pitman SG. J Appl Phys 2012;112:043511.

[6] Verhelst S, Wallner T. Prog Energy Combust Sci 2009;35:490.

[7] Shafiei a., Oprea C, Alfantazi a., Troczynski T. J Appl Phys 2011;109:114108.

[8] Warusawithana MP, Cen C, Sleasman C, Woicik JC, Li Y. Science (80- ) 2009;324:367.

[9] Aggarwal S, Perusse SR, Nagaraj B, Ramesh R. Appl Phys Lett 1999;74:3023.

[10] Kreuer K. Solid State Ionics 1999;125:285.

[11] Münch W, Kreuer K, Seifert G, Maier J. Solid State lonics 2000;137:183.

[12] Huang HY, Chu WY, Su YJ, Gao KW, Li JX, Qiao LJ. J Am Ceram Soc 2007;90:2062.

[13] Kaneda K, Lee S, Donnelly NJ, Qu W, Randall CA, Mizuno Y. J Am Ceram Soc 2011;94:3934.

[14] Waser R. Berichte der Bunsengesellschaft für Phys Chemie 1986;90:1223.

[15] Alvine KJ, Tyagi M, Brown CM. J Appl Phys 2012;111:053505.

[16] Han J, Ma T. Appl Phys Lett 1997;71:1267.

[17] Lvovich VF. Impedance Spectroscopy: Applications to Electrochemical and Dielectric Phenomena. Hoboken: Wiley; 2012.

[18] Barsoukov E, Macdonald JR. Impedance Spectroscopy: Theory, Experiment, and Applications, 2nd Edition. Hoboken: Wiley; 2005.

[19] Yang GY, Lian GD, Dickey EC, Randall C a., Barber DE, Pinceloup P, Henderson M a., Hill R a., Beeson JJ, Skamser DJ. J Appl Phys 2004;96:7500.

[20] Chazono H, Kishi H. Jpn J Appl Phys 2001;40:5624.

[21] Sinclair DC, West a. R. J Appl Phys 1989;66:3850.

[22] Donnelly NJ, Randall CA. J Am Ceram Soc 2009;92:405.

[23] Chan N, Smyth D. J Am Ceram Soc 1984;67:285.

[24] Chan H, Harmer MR, Smyth DML. J Am Ceram Soc 1986;10:507. 
[25] Schroder DK. Semiconductor Material and Device Characterization. New York: John Wiley; 1998.

[26] Sohrabi Baba Heidary D, Qu W, Randall C a. J Appl Phys 2015;117:124104.

[27] Opitz AK, Lutz A, Kubicek M, Kubel F, Hutter H, Fleig J. Electrochim Acta 2011;56:9727.

[28] Shewmon P. Diffusion in Solids. Hoboken: Wiley; 1991.

[29] Donnelly NJ, Randall C a. IEEE Trans Ultrason Ferroelectr Freq Control 2012;59:1883.

[30] Crank J. The Mathematics of Diffusion, Second Edition. London: Oxford University Press; 1975.

[31] Mehrer H. Diffusion in Solids:Fundamentals, Methods, Materials, Diffusion-Controlled Processes. Berlin: Springer; 2007.

[32] Shaw D. Atomic Diffusion in Semiconducters. New York: Plenum Press; 1973.

[33] Norby T, Widerøe M, Glöckner R, Larring Y. Dalt Trans 2004:3012.

[34] Münch W, Kreuer K, Seifertli G, Majer J. Solid State Ionics 1999;125:39.

[35] Giannici F, Longo A, Balerna A, Kreuer K-D, Martorana A. Chem Mater 2009;21:2641.

[36] Yamazaki Y, Blanc F, Okuyama Y, Buannic L, Lucio-Vega JC, Grey CP, Haile SM. Nat Mater 2013;12:647.

[37] Karlsson M, Engberg D, Björketun ME, Matic A, Wahnström G, Sundell PG, Berastegui P, Ahmed I, Falus P, Farago B, Börjesson L, Eriksson S. Chem Mater 2010;22:740.

[38] Naundorf V, Macht M, Bakai A, Lazarev N. J Non Cryst Solids 1998;252:679.

[39] Naundorf V, Macht M, Bakai A, Lazarev N. J Non Cryst Solids 1998;224:122.

[40] Kirchheim R, Sommer F, Schluckebier G. Acta Metall 1982;30:1059.

[41] Kirchheim R. Acta Metall 1982;30:1069.

[42] Sze SM, Kwok KN. Physics of Semiconductor Devices. Hoboken: John Wiley \& Sons; 2007.

[43] Skriver H, Rosengaard N. Phys Rev B 1992;46:7157.

[44] Wilson RG. J Appl Phys 1966;37:2261.

[45] Dweydari A, Mee C. Phys status solidi 1975;27:223.

[46] Uda M, Nakamura A. J Electron Spectros Relat Phenomena 1998;91:643. 
[47] Lide DR. CRC Handbook of Chemistry and Physics, 89 Edition. Boca Raton: CRC Press; 2008.

[48] Peacock PW, Robertson J. J Appl Phys 2002;92:4712.

[49] Cann D, Randall C. IEEE Trans Ultrason Ferroelectr Freq Control 1997;44:1405.

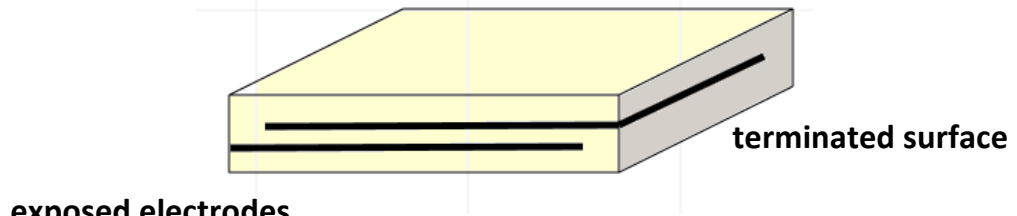

exposed electrodes

Figure 1 the schematic of cut capacitors. 


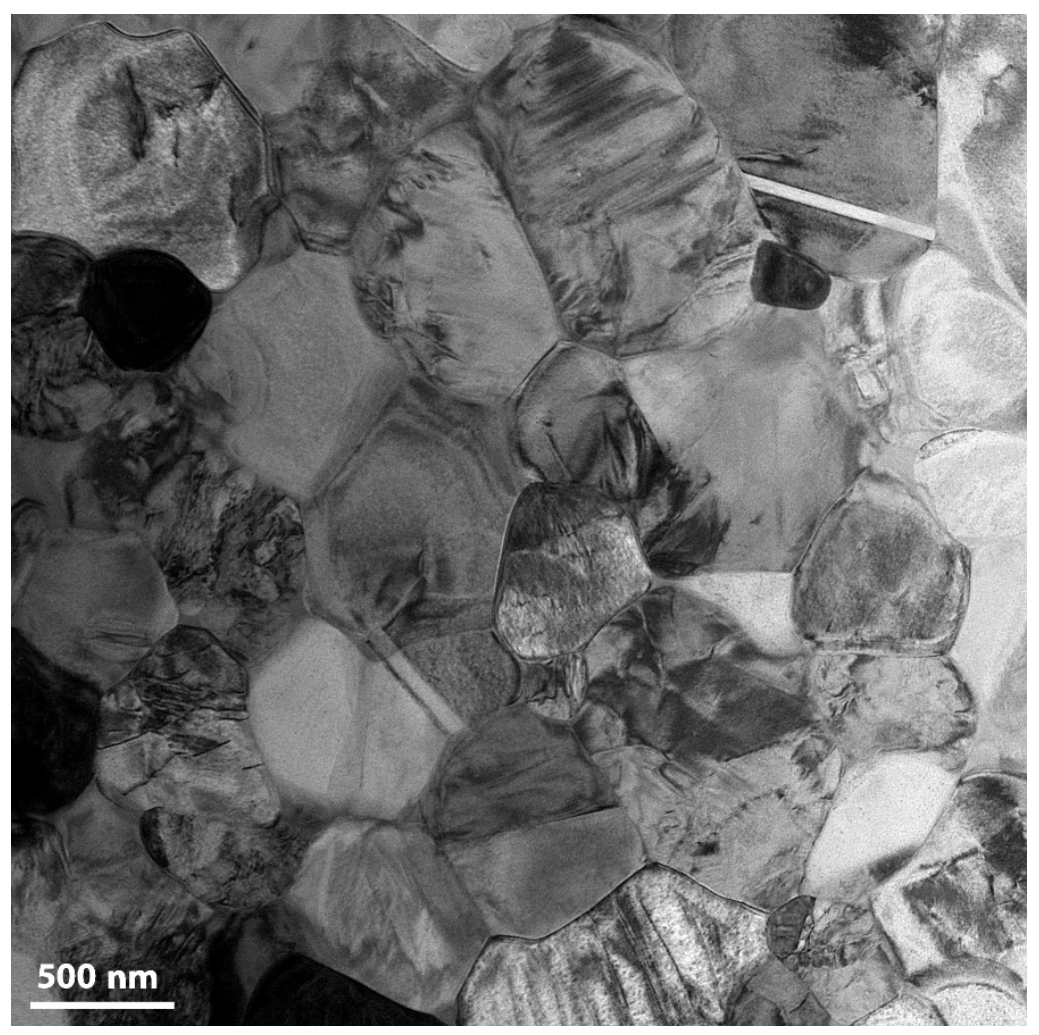

Figure 2 TEM micrograph of sintered $\mathrm{BaTiO}_{3}$ dielectric microstructure. Grains have domain structures and, in some cases, $\{111\}$ growth twins; average grain size is about 0.5 microns. 

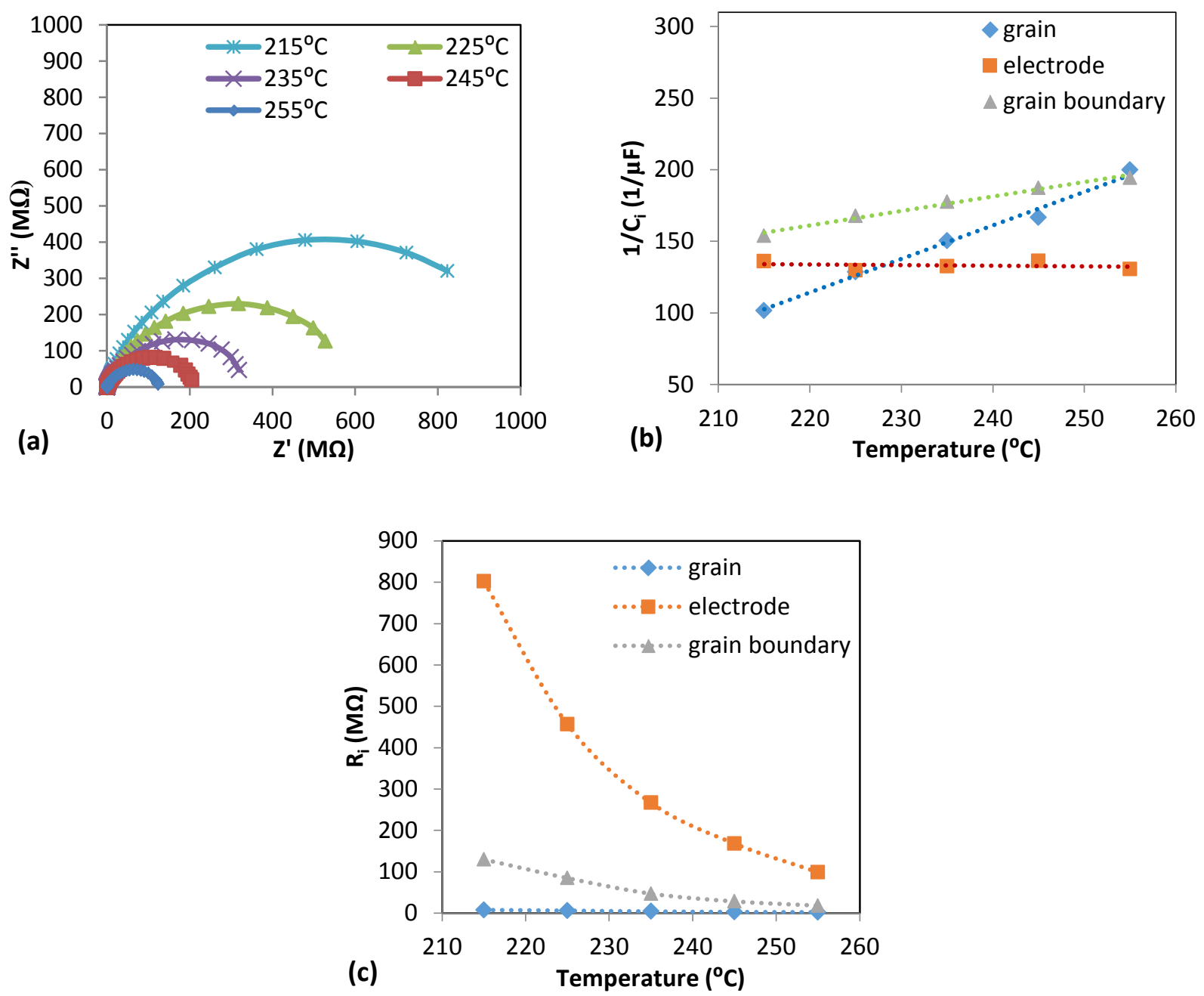

Figure 3 (a) Imaginary impedance (z’') vs. real impedance (z') at different temperatures for the $\mathrm{BaTiO}_{3}$ single layer capacitor structure, (b) 1/C $\mathrm{i}$ vs temperature, and (c) resistivity vs. temperature based on the 3RC model and Cole-Cole graphs in (a). 


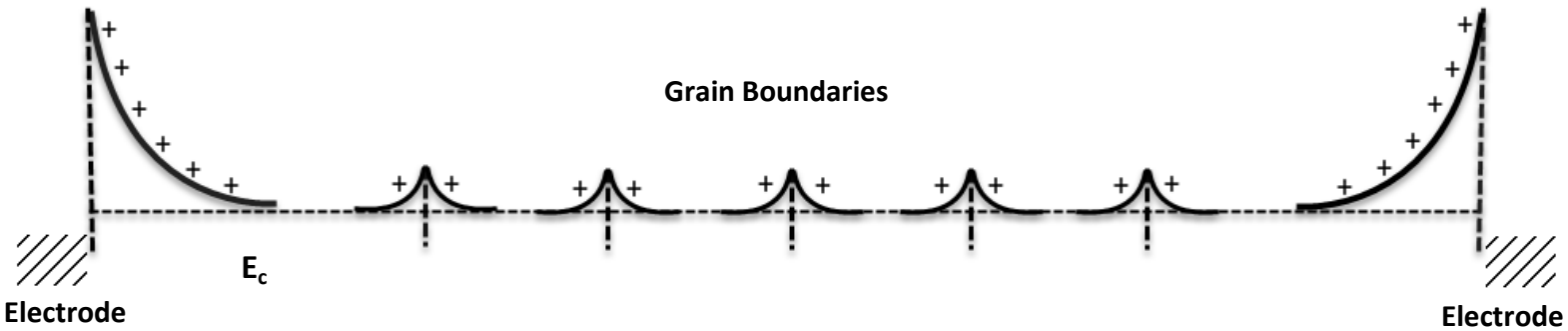

Figure 4 A schematic of electrode and grain boundary Schottky barriers in the capacitor active layer under no external fields. 

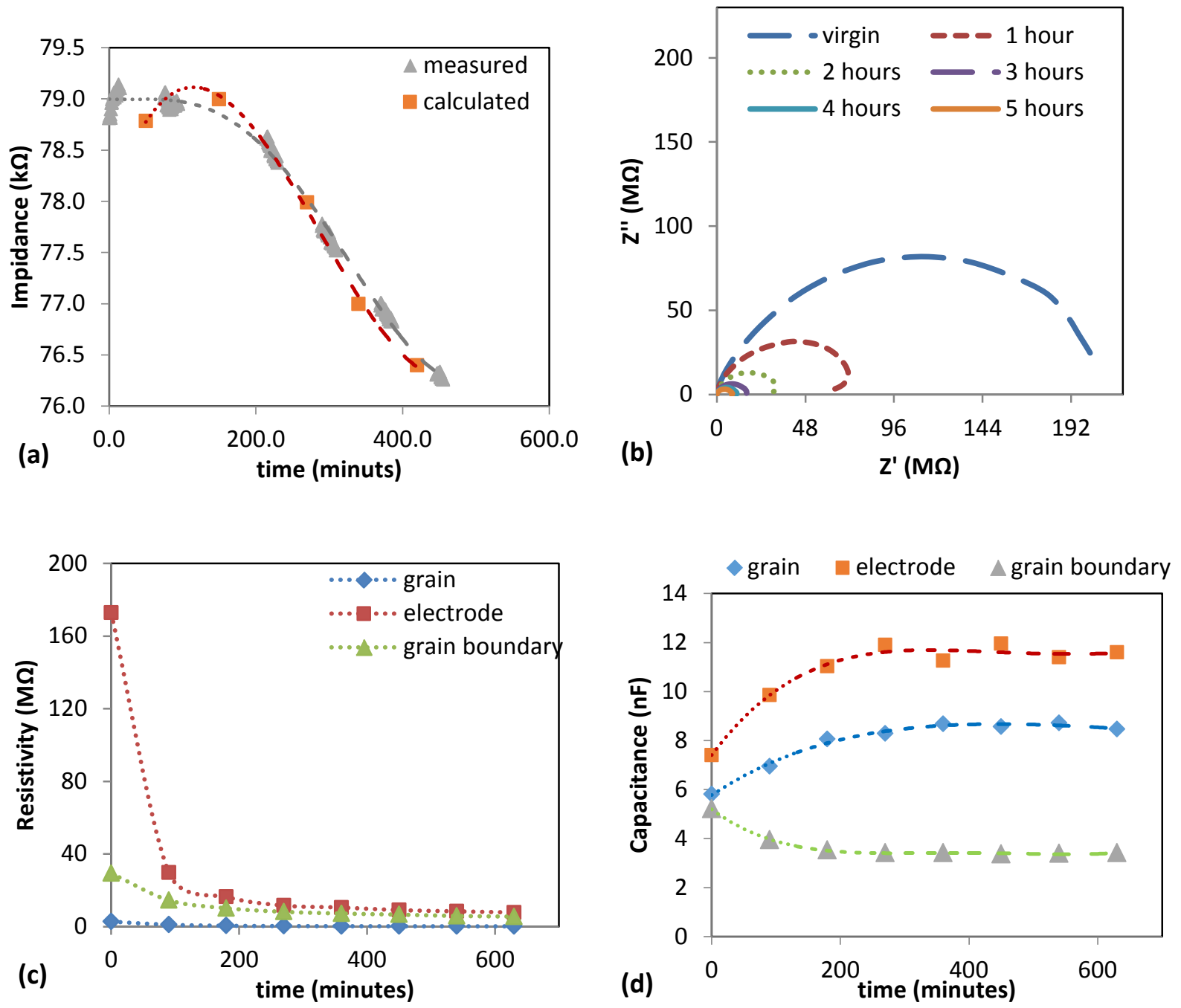

Figure 5 (a) Measured and calculated impedance at $1 \mathrm{kHz}$ vs exposure time to hydrogen gas. (b) Imaginary impedance $\left(z^{\prime \prime}\right)$ vs real impedance (z') at different exposure time at $245^{\circ} \mathrm{C}$. The result of $3 \mathrm{RC}$ model, separated to ideal (c) resistors and (d) capacitors vs. time based on data on the Cole-Cole plots in (b). 


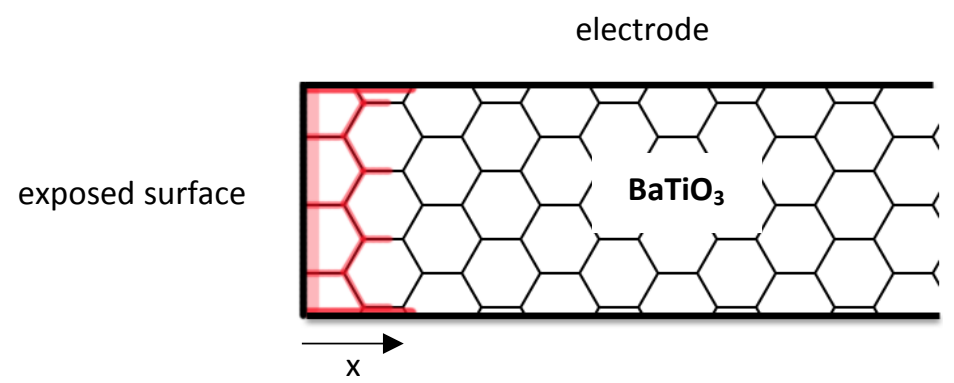

Figure 6 Schematic of hydrogen diffusion in BME capacitors. The red ink shows the hydrogen cations. The difference between diffusion rates in electrode interfaces, grain boundaries, and grains are drawn proportionally. 

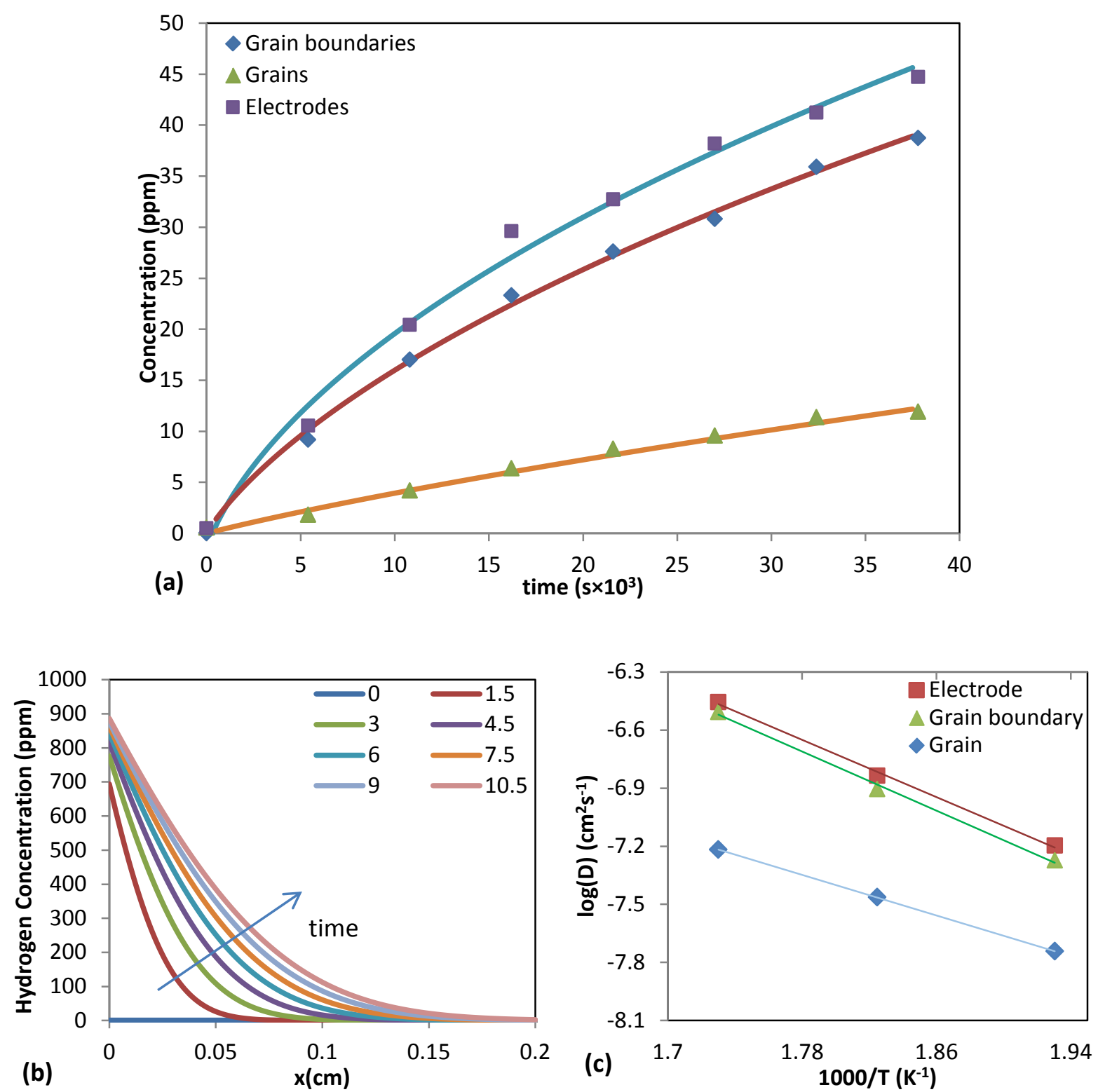

Figure 7. (a) Hydrogen concentration vs. time for $\mathrm{BaTiO}_{3}$ buried layer capacitor at $245^{\circ} \mathrm{C}$ at three $\mathrm{RC}$ components. (b) Distribution of hydrogen impurities along the electrode- $\mathrm{BaTiO}_{3}$ interface at every 1.5 hours after exposing to hydrogen gas. (c) Diffusion coefficients of hydrogen at three RC components vs inverse of temperature. 


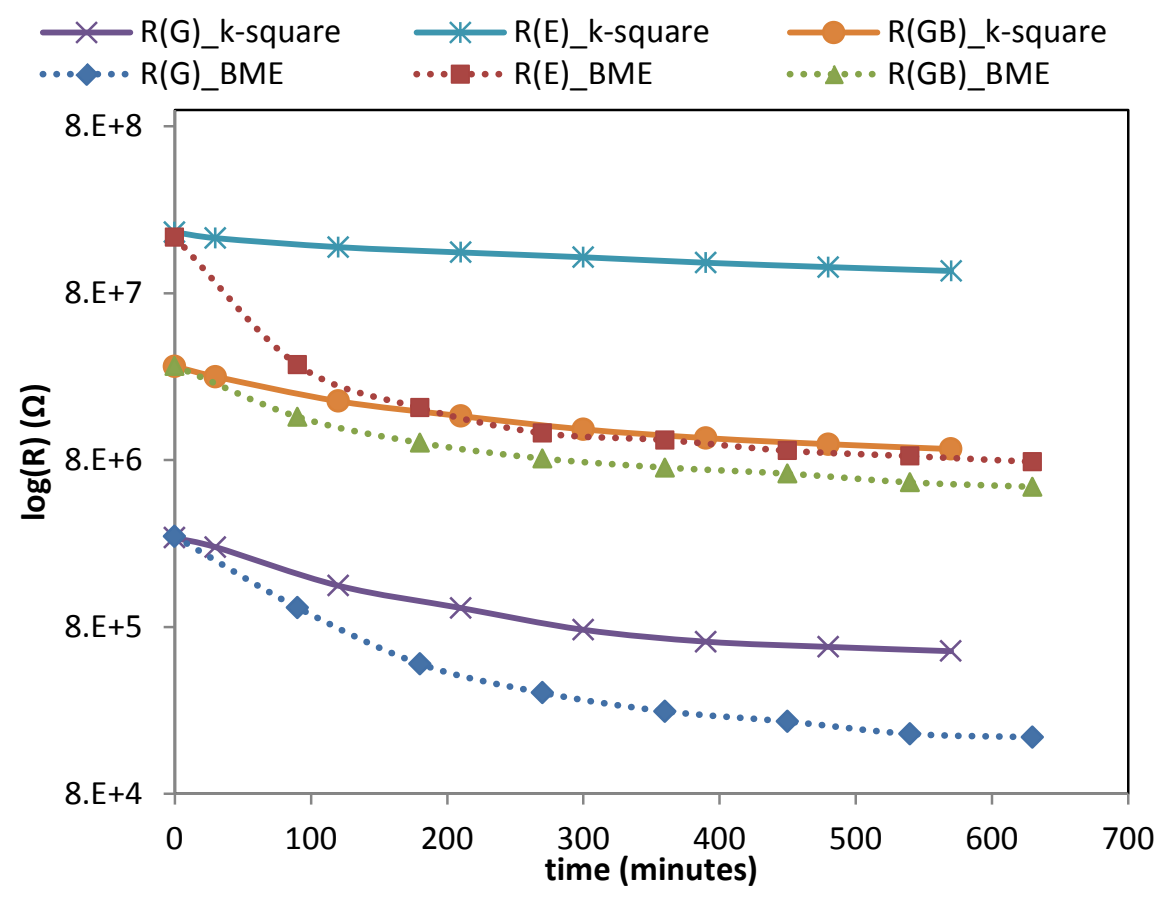

Figure 8 Resistivity of electrode, grain boundary, and grain components vs. time at hydrogen atmosphere for BME and ksquare samples. 

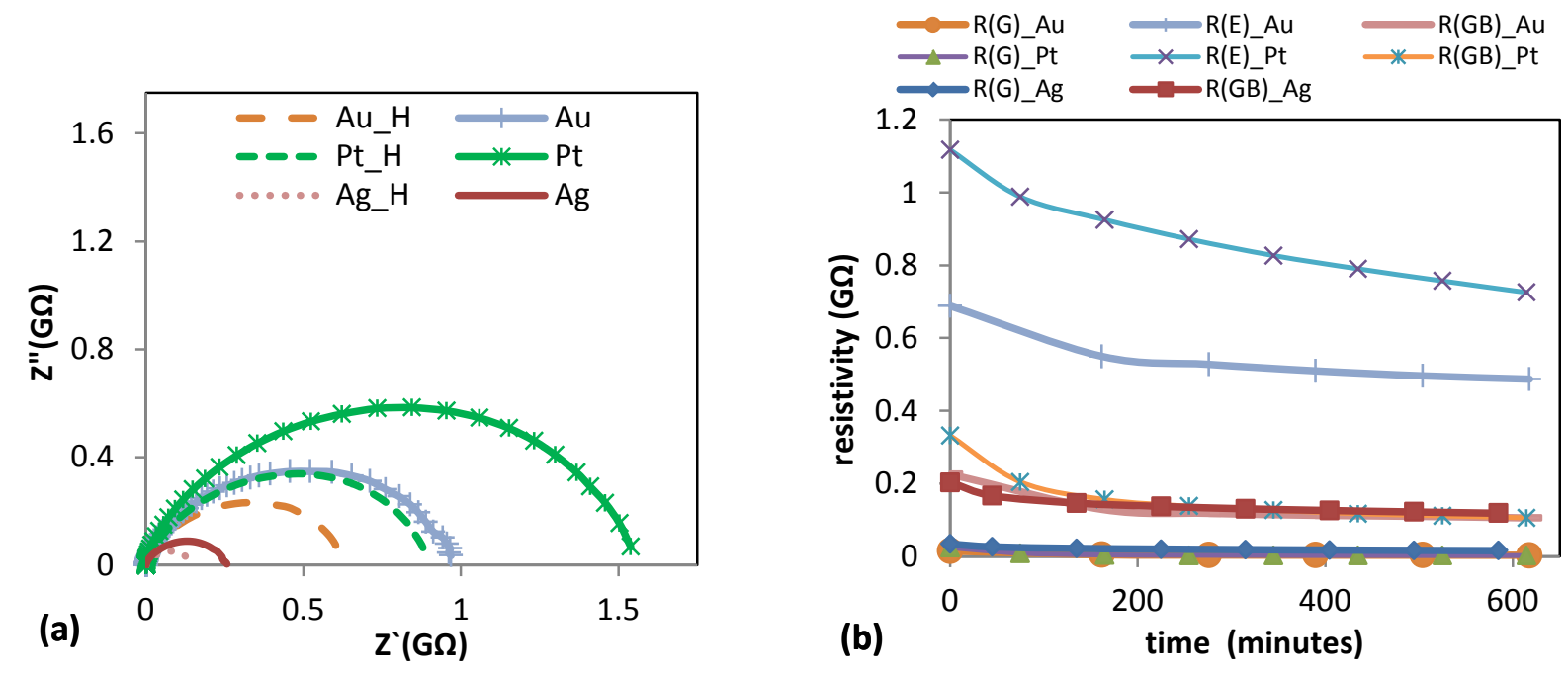

Figure 9. Impedance result of the k-square samples with silver, platinum, gold electrodes at $245^{\circ} \mathrm{C}$. (a) Cole-Cole plot before and after exposing to hydrogen for 10 hours, and (b) calculated resistivity vs. time for electrode, grains, and grain boundary for each sample. 
Table 1 Diffusion behavior of $\mathrm{BaTiO}_{3}$ capacitor in electrodes, grains, and grain boundaries.

\begin{tabular}{|l|c|c|c|}
\hline & $\begin{array}{c}\text { Diffusion coefficient (D) } \\
\text { at } 240^{\circ} \mathrm{C}\left(\mathrm{cm}^{-2} \mathrm{~s}^{-1}\right)\end{array}$ & $\begin{array}{c}\mathrm{D}_{0} \text { pre-exponential } \\
\text { factor }\left(\mathrm{cm}^{-2} \mathrm{~s}^{-1}\right)\end{array}$ & $\begin{array}{c}\mathrm{E}_{\mathrm{A}} \text { activation enthalpy } \\
(\mathrm{eV})\end{array}$ \\
\hline Electrodes & $6.35 \times 10^{-8}$ & 0.8788 & 0.73 \\
\hline Grain Boundaries & $5.32 \times 10^{-8}$ & 1.2779 & 0.76 \\
\hline Grains & $1.80 \times 10^{-8}$ & $2.02 \times 10^{-3}$ & 0.52 \\
\hline
\end{tabular}


Table 2. List of activation enthalpy and pre-exponential factor for bulk diffusion at various perovskite oxides.

\begin{tabular}{|l|c|c|c|}
\hline Oxide & crystal structure & $\mathrm{D}_{0}\left(\mathrm{~cm}^{2} / \mathrm{s}\right)$ & $\mathrm{E}_{\mathrm{a}}(\mathrm{eV})$ \\
\hline $\mathrm{Ba}\left(\mathrm{Zr}_{0.9} \mathrm{Y}_{0.1}\right) \mathrm{O}_{3}$ & Cubic & $2.34 \times 10^{-3}$ & $0.43[10]$ \\
\hline $\mathrm{Sr}\left(\mathrm{Ti}_{0.95} \mathrm{Sc}_{0.05}\right) \mathrm{O}_{3}$ & Cubic & $7.41 \times 10^{-4}$ & $0.42[10]$ \\
\hline $\mathrm{Ba}\left(\mathrm{Ti}_{0.95} \mathrm{Sc}_{0.05}\right) \mathrm{O}_{3}$ & Cubic & $1.95 \times 10^{-2}$ & $0.64[10]$ \\
\hline $\mathrm{Pb}(\mathrm{Zr}, \mathrm{Ti}) \mathrm{O}_{3}$ & Cubic & - & $0.44[7]$ \\
\hline $\mathrm{CaTiO3}$ & Cubic & $1.48 \times 10^{-3}$ & $0.42[34]$ \\
\hline $\mathrm{SrTiO}{ }_{3}$ & Cubic & $1.12 \times 10^{-3}$ & $0.5[34]$ \\
\hline $\mathrm{Ba}\left(\mathrm{Zr}_{98} \mathrm{In}_{2}\right) \mathrm{O}_{3}$ & Cubic & $2.2 \times 10^{-4}$ & $0.45[35]$ \\
\hline $\mathrm{Ba}\left(\mathrm{Zr}_{50} \mathrm{In}_{50}\right) \mathrm{O}_{3}$ & Cubic & $1.1 \times 10^{-4}$ & $0.62[35]$ \\
\hline $\mathrm{Sr}\left(\mathrm{Ce}_{0.9} \mathrm{Y}_{0.1}\right) \mathrm{O}_{3}$ & non-cubic & $8.32 \times 10^{-3}$ & $0.63[10]$ \\
\hline $\mathrm{Ba}\left(\mathrm{Ce}_{0.85} \mathrm{Y}_{0.15}\right) \mathrm{O}_{3}$ & non-cubic & $3.47 \times 10^{-2}$ & $0.6[10]$ \\
\hline $\mathrm{BaZrO}{ }_{3}$ & Cubic & $3 \times 10^{-5}$ & $0.17-0.20[36][37]$ \\
\hline $\mathrm{Ba}\left(\mathrm{Zr}_{0.8} \mathrm{Y}_{0.2}\right) \mathrm{O}_{3}$ & & $5 \times 10^{-2}$ & $0.47[36]$ \\
\hline
\end{tabular}

